\title{
THE HYBRID CAPRICCIO METHOD: A 1D STUDY FOR FURTHER ADVANCEMENT
}

\author{
Maximilian Ries ${ }^{1}$, Paul Steinmann $^{2}$ and Sebastian Pfaller $^{3}$ \\ Institute of Applied Mechanics \\ Friedrich-Alexander University Erlangen-Nürnberg \\ Egerlandstr. 591058 Erlangen, Germany \\ ${ }^{1}$ maximilian.ries@fau.de \\ 2 paul.steinmann@fau.de \\ 3 sebastian.pfaller@fau.de
}

Key words: particle-continuum coupling, domain decomposition, multiscale-modeling

\begin{abstract}
Polymers and, in particular, polymer composites are known for the enormous adjustability of their mechanical, chemical, and thermal behavior. Multiscale methods are increasingly employed to unravel the polymer microstructure's impact on the material properties. These methods combine the accuracy of particle-based techniques with the efficiency of continuum mechanical approaches. Amorphous polymers pose a special challenge since their microstructure does not continue periodically, and therefore special attention needs to be paid to the particle domain boundary.

In this study, we introduce a coupling via an interface between the continuum and the particle domain. Padding atoms as particle representations of the continuum, which serve as interaction partners for the atoms in the particle region, allow for the transfer of displacements and forces between the domains. We present a straightforward 1D example with simple interactions, evaluate the scheme's performance, discuss the resulting energy contributions, and identify an optimal set of coupling parameters. Eventually, this forms the basis for future 3D implementations.
\end{abstract}

\section{INTRODUCTION}

Polymers are an outstanding material since they are typically inexpensive, lightweight, and versatile. In particular, they can be adapted to numerous applications' requirements, e.g., by adding fillers. Particlebased numerical methods are often used to support the development of such composites. However, the enormous computational effort required to investigate sufficiently large samples limits their use substantially. Therefore, multiscale approaches are employed in many cases, using the accuracy of particle-based methods only where necessary and relying on a coarser description in the remaining regions. Recently, Zhao et al. [1] have introduced a continuum mechanical constitutive law that is capable of reproducing the mechanical behavior of polymers obtained from molecular dynamics simulations [2, 3].

A particle-continuum coupling has been realized for crystalline materials most prominently with the Quasicontinuum method [4, 5], the Arlequin method [6, 7] or the Bridging Domain method [8, 9]. Tad- 
mor et al. [10] give a thorough overview of these approaches and a comprehensive comparison can be found in [11]. Pfaller et al. incorporated the preceding methods' ideas and developed the Capriccio method $[12,13,14,15,16]$ for the treatment of amorphous polymers. The coupling of the particle and the continuum domains is realized within a hand-shake region by introducing anchor points. These virtual particles transfer information between the two resolutions and facilitate the particle and continuum displacement fields to match. The Capriccio method has proven its capabilities in investigating mechanical interphases in polymer nanocomposites $[17,18]$. Nevertheless, there remain artifacts of the coupling, most likely rooting within the hand-shake region.

In this study, we evaluate the implications of a less complicated coupling scheme via an interface and whether this approach can be suitable for further developing the Capriccio method. To this end, we introduce a straightforward 1D example with simple interactions, discuss the resulting energy contributions, and identify an optimal set of coupling parameters.

\section{1D SETUP}

We consider the simplest possible one-dimensional system to study the coupling of a continuum domain $\Omega_{c}$ and a particle region $\Omega_{d}$ along a boundary surface. Polymers are usually modeled by local and nonlocal interactions, while continuum mechanics is a purely local method. To take this mismatch into account, we consider the next-neighbor (nn) and second-next-neighbor (sn) interactions

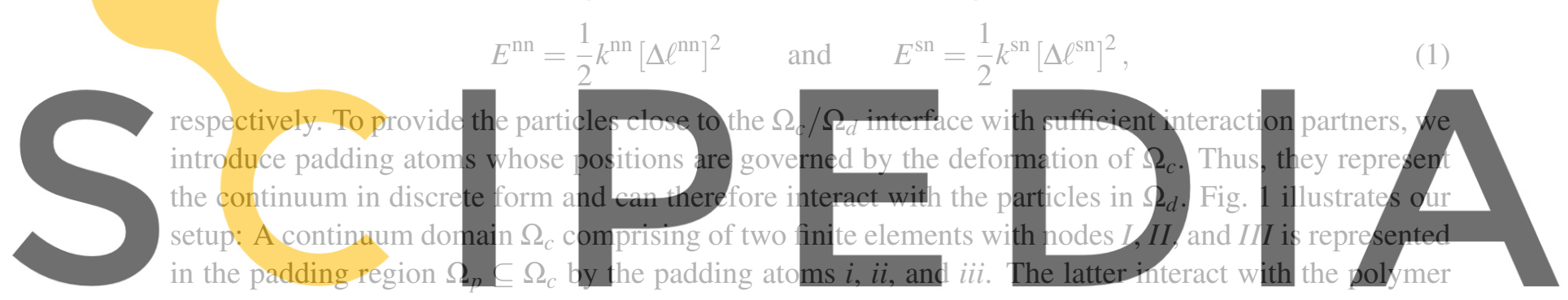

chain's atoms 1,2 , and 3 , thus mimicking the polymer chain's continuation within $\Omega_{C}$.

Register for free at https//www.scipedią.com to download thę version without the watermark

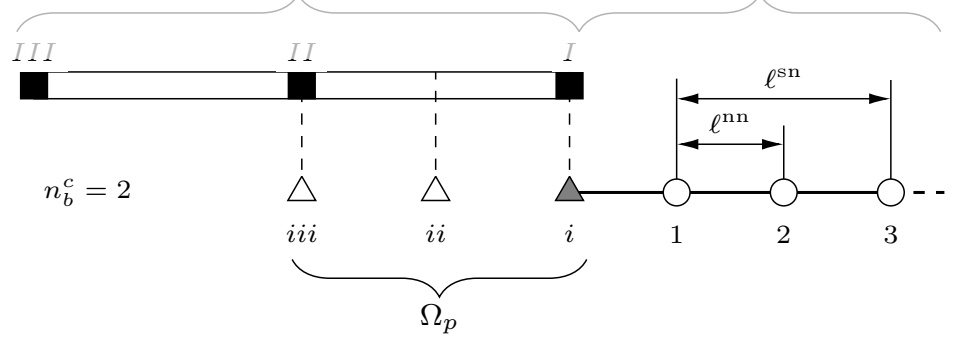

Figure 1: 1D setup: continuum domain $\Omega_{c}$ comprising two finite elements with nodes $I, I I$, and $I I I$; particle domain $\Omega_{d}$ with a polymer chain of atoms 1,2 , and 3; interaction partners for $\Omega_{d}$ are provided by padding atoms $i, i i$, and $i i i$ in the padding region $\Omega_{p}$.

From $k^{\mathrm{nn}}$ and $k^{\mathrm{sn}}$ we derive the equivalent stiffness of a finite element

$$
k^{\mathrm{c}}=\frac{1}{n_{b}^{\mathrm{c}}}\left[k^{\mathrm{nn}}+4 k^{\mathrm{sn}}\right]
$$


containing $n_{b}^{\mathrm{c}}$ next-neighbor bonds. In the system in Fig. 1 we consider $n_{b}^{\mathrm{c}}=2$ and thus $k^{\mathrm{c}}=\frac{1}{2} k^{\mathrm{nn}}+2 k^{\mathrm{sn}}$. In this work's scope, we focus only on time-independent behavior and thus exclude any dynamic effects.

The positions of particles $i i i$ and $i$ coincide with nodes $I$ and $I I$, while particle $i i$ is supposed to mark the middle of the element, hence

$$
\left[\begin{array}{c}
u_{i} \\
u_{i i} \\
u_{i i i}
\end{array}\right]=\left[\begin{array}{c}
u_{I} \\
0.5\left[u_{I}-u_{I I}\right] \\
u_{I I}
\end{array}\right]
$$

holds for the displacements.

Interface atom $i$ (cf. Fig. 1) is energetically treated as a regular atom, but its position is predefined by FE node $I$. To account for the contribution of $i$ and thus avoid a double-counting of particle and continuum energies, we introduce the weighting factor

$$
\alpha_{c}=1-\frac{1}{2 n_{b}^{c}},
$$

yielding $\alpha_{c}=\frac{3}{4}$ for $n_{b}^{c}=2$. As a result, we obtain the weighted stiffness matrix of the continuum domain

$$
\boldsymbol{K}^{\mathrm{c}}=\left[\begin{array}{ll}
\boldsymbol{K}_{F F}^{\mathrm{c}} & \boldsymbol{K}_{F P}^{\mathrm{c}} \\
\boldsymbol{K}_{P F}^{\mathrm{c}} & \boldsymbol{K}_{P P}^{\mathrm{c}}
\end{array}\right]=\left[\begin{array}{c|cc}
-1 & 1 & 0 \\
\hline 1 & -\left[1+\alpha_{c}\right] & \alpha_{c} \\
0 & \alpha_{c} & -\alpha_{c}
\end{array}\right] k^{\mathrm{c}}
$$

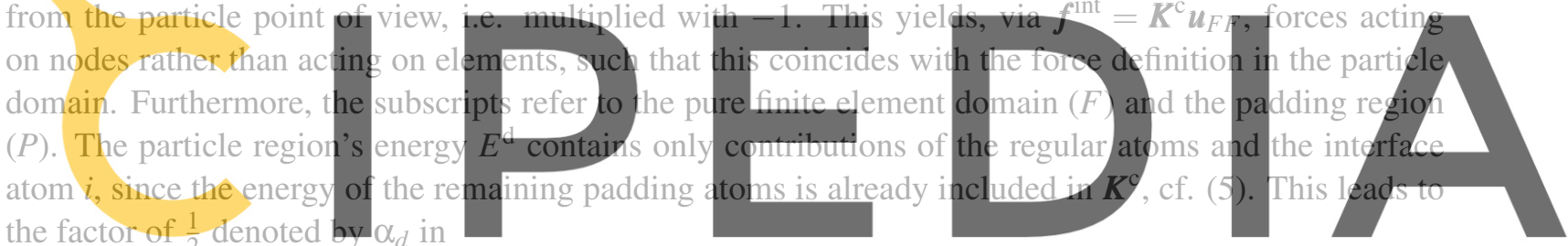
the factor of $\frac{1}{2}$ denoted by $\alpha_{d}$ in

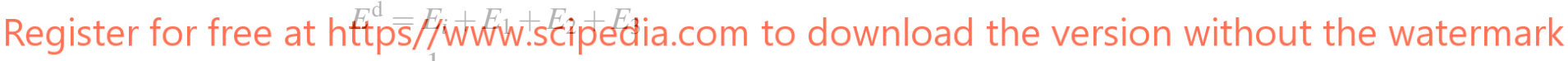

$$
=\frac{1}{2} \underbrace{\left[E_{i i i}+E_{i i i i}+E_{1 i i}\right]}_{E_{P A}}+\underbrace{E_{i 1}+E_{i 2}+E_{12}+E_{13}+E_{23}}_{E_{A A}}=\alpha_{d} E_{P A}+E_{A A}
$$

with the energy $E_{k l}$ due to interaction of particles $k$ and $l \in \Omega_{p} \cup \Omega_{d}$ and the subscripts referring to the padding region $(P)$ and the pure atomistic domain $(A)$.

The particle stiffness matrix can thus be written as $\boldsymbol{K}^{\mathrm{d}}=\left[\begin{array}{ll}\boldsymbol{K}_{P P}^{\mathrm{d}} & \boldsymbol{K}_{P A}^{\mathrm{d}} \\ \boldsymbol{K}_{A P}^{\mathrm{d}} & \boldsymbol{K}_{A A}^{\mathrm{d}}\end{array}\right]=$

$=\left[\begin{array}{ccc|ccc}-\alpha_{d} k^{\mathrm{sn}}-k^{\mathrm{nn}} & k^{\mathrm{nn}} & \alpha_{d} k^{\mathrm{sn}} & 0 & 0 & 0 \\ k^{\mathrm{nn}} & -\alpha_{d} k^{\mathrm{sn}}\left[1+\alpha_{d}\right] k^{\mathrm{nn}} & \alpha_{d} k^{\mathrm{nn}} & \alpha_{d} k^{\mathrm{sn}} & 0 & 0 \\ \alpha_{d} k^{\mathrm{sn}} & \alpha_{d} k^{\mathrm{nn}} & -\left[\alpha_{d}+1\right]\left[k^{\mathrm{sn}}+k^{\mathrm{nn}}\right] & k^{\mathrm{nn}} & k^{\mathrm{sn}} & 0 \\ \hline 0 & \alpha_{d} k^{\mathrm{sn}} & k^{\mathrm{nn}} & -\left[\alpha_{d}+1\right] k^{\mathrm{sn}}-2 k^{\mathrm{nn}} & k^{\mathrm{nn}} & k^{\mathrm{sn}} \\ 0 & 0 & k^{\mathrm{sn}} & k^{\mathrm{nn}} & -k^{\mathrm{sn}}-2 k^{\mathrm{nn}} & k^{\mathrm{nn}} \\ 0 & 0 & 0 & k^{\mathrm{sn}} & k^{\mathrm{nn}} & -k^{\mathrm{sn}}-k^{\mathrm{nn}}\end{array}\right]$.


Note that in a pure particle setting, the weighting factors $\alpha_{d}$ highlighted in red would not be present, indicating that $\alpha_{d} \neq 1$ attributes to missing stiffness contributions in this coupling scheme. Since these missing entries are part of $\boldsymbol{K}_{P A}^{\mathrm{d}}, \boldsymbol{K}_{A P}^{\mathrm{d}}$, and $\boldsymbol{K}_{A A}^{\mathrm{d}}$, respectively, they cannot be compensated by the continuum which is acting only locally, and thus can only contribute to $\boldsymbol{K}_{P P}^{\mathrm{d}}$.

Evaluating the shape functions $N_{\eta=I}$ and $N_{\eta=I I}$ of nodes $I$ and $I I$ at the position $r_{j}$ of each padding atom $j \in \Omega_{p}$ yields the coupling matrix

$$
\boldsymbol{G}_{w u}=\left[\begin{array}{cc}
N_{\eta=I}\left(r_{j}\right) & N_{\eta=I I}\left(r_{j}\right) \\
\vdots & \vdots \\
N_{\eta=I}\left(r_{n_{P}^{c}}\right) & N_{\eta=I I}\left(r_{n_{P}^{c}}\right)
\end{array}\right]
$$

which simplifies in our case to

$$
\boldsymbol{G}_{w u}=\left[\begin{array}{cc}
1 & 0 \\
0.5 & 0.5 \\
0 & 1
\end{array}\right]
$$

Finally, this enables us to assemble our system of equations

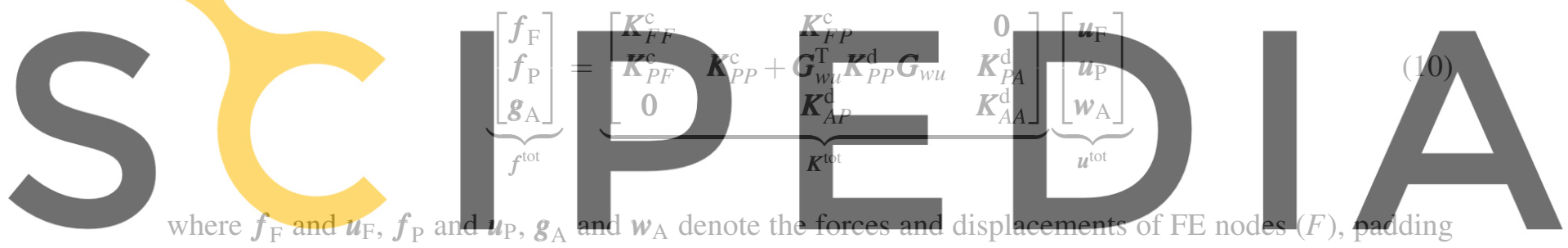
atoms $(P)$, and regular atoms $(A)$, respectively.

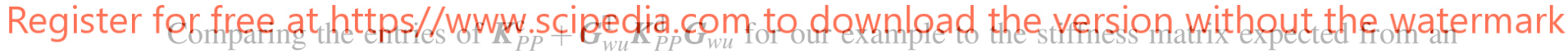
infinite particle chain $\overline{\boldsymbol{K}}^{\mathrm{d}}$ results in the deviation

$$
\Delta \boldsymbol{K}=\overline{\boldsymbol{K}}^{\mathrm{d}}-\boldsymbol{K}_{P P}^{\mathrm{c}}+\boldsymbol{G}_{w u}^{\mathrm{T}} \boldsymbol{K}_{P P}^{\mathrm{d}} \boldsymbol{G}_{w u}=\left[\begin{array}{ccc}
\frac{3}{2} k^{\mathrm{sn}}-\frac{1}{4} k^{\mathrm{nn}} & -3 k^{\mathrm{sn}}+\frac{1}{4} k^{\mathrm{nn}} & \frac{1}{2} k^{\mathrm{sn}} \\
-3 k^{\mathrm{sn}}+\frac{1}{4} k^{\mathrm{nn}} & \frac{9}{2} k^{\mathrm{sn}} & -3 k^{\mathrm{sn}}-\frac{1}{4} k^{\mathrm{nn}} \\
\frac{1}{2} k^{\mathrm{sn}} & -3 k^{\mathrm{sn}}-\frac{1}{4} k^{\mathrm{nn}} & \frac{5}{2} k^{\mathrm{sn}}+\frac{1}{4} k^{\mathrm{nn}}
\end{array}\right] .
$$

It is obvious, that the parameters $\alpha_{d}=\frac{1}{2}$ and $\alpha_{c}=\frac{3}{4}$, cf. (6) and (4), respectively, will not result in an ideal coupling. Therefore, we aim to determine an optimized set of weighting factors in the following section.

\section{PARAMETER IDENTIFICATION}

For the following numerical analyses, we implement the proposed coupling scheme in Matlab (R2016b) in a unitless way. As boundary conditions for our example system (cf. Fig. 1), we fix the FE node on the left side and apply a force $F=1$ to the rightmost atom. As depicted in Fig. $1, \Omega_{p}$ contains one padding element $n_{P}^{\mathrm{c}}$ which represents two bonded interactions $n_{b}^{\mathrm{c}}$ such that three padding atoms are required. To 
avoid a perturbation of the coupling by boundary effects, we enlarge our system and consider 4 pure FE elements $n_{\mathrm{FE}}^{\mathrm{c}}$, and 10 regular atoms $n_{A}^{\mathrm{d}}$ in $\Omega_{d}$. We set $k^{\mathrm{nn}}=2$ and $k^{\mathrm{sn}}=1$ for the particle interactions, hence $k^{\mathrm{c}}=3$ according to (2).

The resulting displacements of the coupled system, obtained with $\alpha_{c}$ and $\alpha_{d}$ according to (4) and (6), respectively, is depicted in Fig. 2 a). As expected, there is a boundary influence affecting up to 4 atoms at the right end of the chain since the applied force does not represent an infinite chain's interactions. In order to perform a quantitative comparison with the analytic solution (denoted as pure FE), we introduce the error measure

$$
\Delta=\sum_{j \in \Omega_{\Delta}}\left|u_{j}-u_{j}^{\mathrm{FE}}\right|
$$

This is evaluated on $\Omega_{\Delta} \subset\left\{\Omega_{d} \cup \Omega_{b}\right\}$, including the padding atoms and all regular atoms except the last four to omit the surface effects. We are able to find better sets of weighting factors by minimizing the deviation $\Delta$ with the gradient-free Nelder-Mead algorithm [19] (fminsearch) and the gradient-based steepest-decent optimization [20] (1sqcurvefit). These optimized parameters and the obtained displacements and deviations for both algorithms are shown in Fig. 2 b) and c), respectively. As already evident at the end of the previous section, equations (4) and (6) yield a certain deviation $\Delta$. However, through the optimization, we obtain a set of $\alpha_{c}$ and $\alpha_{d}$ that results in a nearly perfect coupling of $\Omega_{c}$ and $\Omega_{d}$.

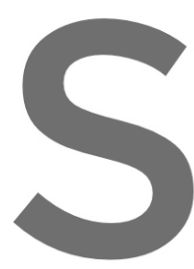

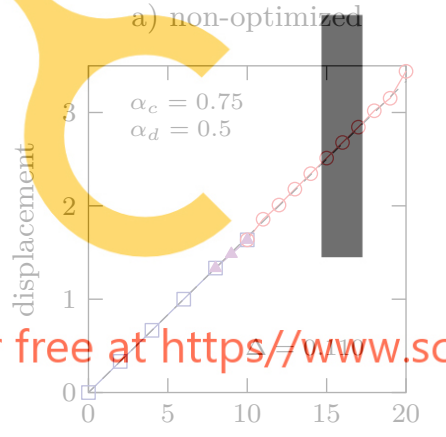

dof
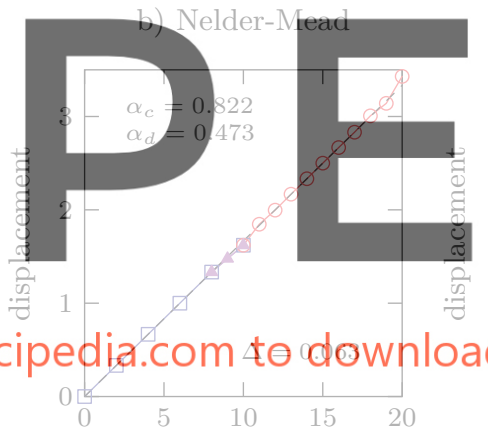

dof
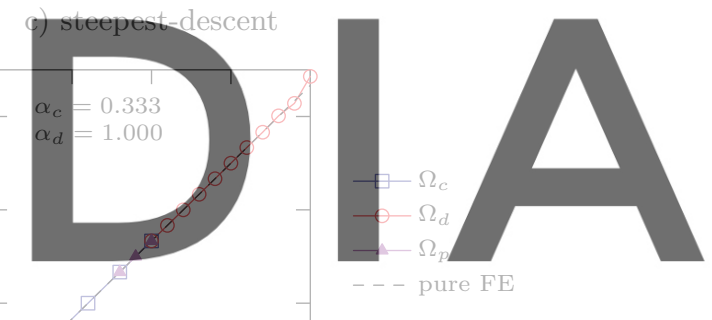

Register for free at https//www.scipedia.com te download the version owithout the watermark

Figure 2: Displacement of coupled systems (cf. Fig. 1) with weighting factors $\alpha_{c}$ and $\alpha_{d}$ obtained by a) equations (4) and (6), b) Nelder-Mead optimization, and c) steepest-decent optimization; deviation $\Delta$ wrt the analytical solution; $n_{A}^{\mathrm{d}}=10, n_{\mathrm{FE}}^{\mathrm{c}}=4, n_{P}^{\mathrm{c}}=1$ and $n_{b}^{\mathrm{c}}=2$.

Next, we would like to gain a better understanding of how to choose these optimal parameters. Even though in Fig. 2 the steepest-decent algorithm leads to better results, in other cases, the Nelder-Mead optimization is superior. Therefore, in the following, we present the least deviation parameter sets attained by either of the two.

Fig. 3 shows the effect of the number of bonds $n_{b}^{\mathrm{c}}$ represented by each padding element on the deviation $\Delta$ for 1,2 , and 3 padding elements. We find a parameter set for each $n_{b}^{\mathrm{c}}$ that leads to negligible deviations by the optimization. The only exception is $n_{b}^{\mathrm{c}}=1$ for 2 and 3 padding elements, resulting in significantly larger $\Delta$. Such a choice, however, would be contradictory to a particle-continuum scale coupling which 
actually aims at large $n_{b}^{\mathrm{c}}$. THus, this case is not further investigated here. Note that the two limit cases of $\Delta=8.7 \cdot 10^{-4}$ and $\Delta=6.8 \cdot 10^{-4}$ (indicated in Fig. 3 by dashed lines) correspond to the steepestdecent and Nelder-mead algorithms, respectively. This means that the former is more robust, while the latter has the potential to perform better, but likely stops in local minima in most cases. For more complex problems, e.g., an extension to 3D, a more thorough investigation should be done on the choice of algorithm and initial values, which is, however, exceeding the scope of this work.

Since it performs best according to Fig. 3 , we focus on the smallest possible padding region $\Omega_{P}$, i.e. $n_{P}^{\mathrm{c}}=1$. Having only one padding element best reflects the idea of coupling across an interface, instead of a handshake region, as used, e.g., in the Capriccio method.
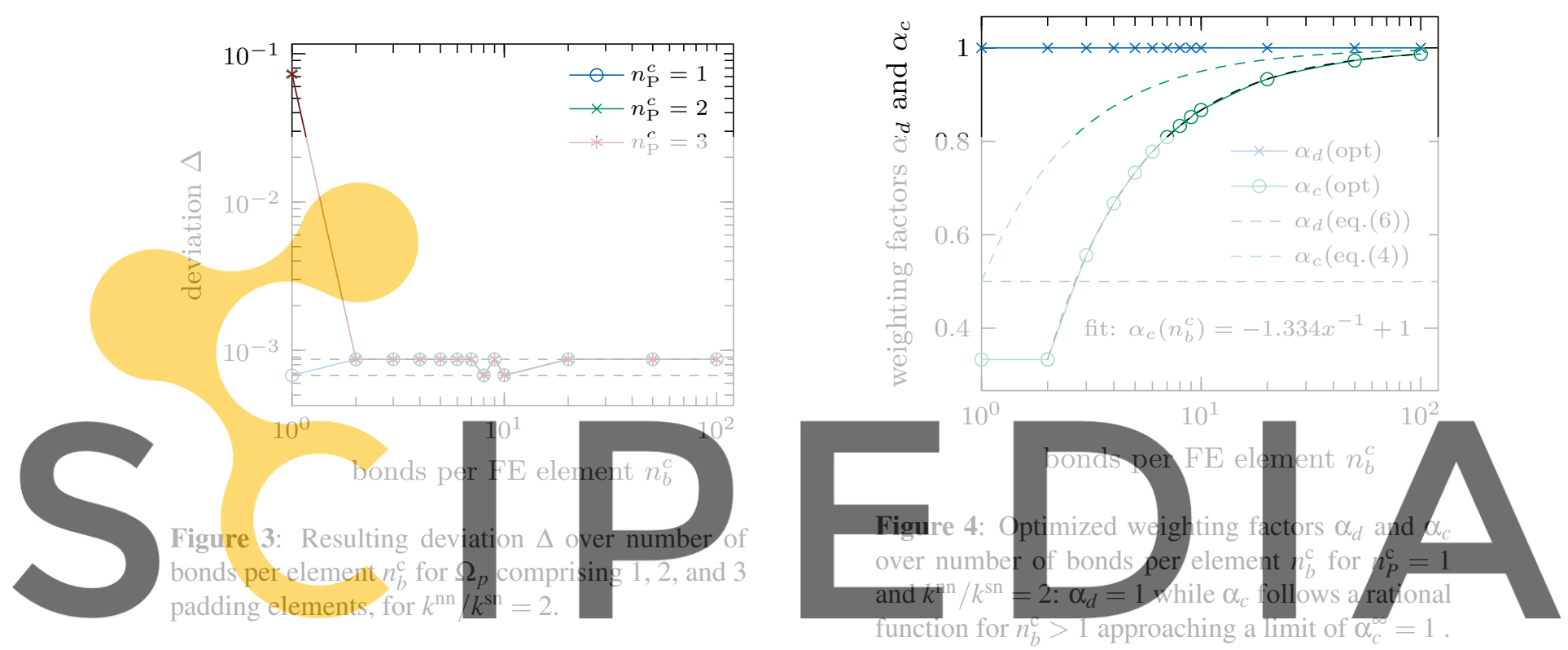

Register for free at https//www.scipedia.com to download the version without the watermark The corresponding sets of weighting factors for the results given in Fig. 3 are presented in Fig. 4 for $n_{P}^{\mathrm{c}}=1$. In contrast to the considerations in (6), the optimizer sets $\alpha_{d}=1$ independent of the number of bonds per padding element. Consequently, all padding atoms contribute to the energy of the particle region

$$
E^{\mathrm{d}}=E_{P A}+E_{A A}
$$

Note that there are no interactions between two padding atoms, since their positions are completely defined by the padding element, hence the finite element accounts for the associated interaction energy and consequently, $E_{P P}=0$ holds. On the contrary, the obtained $\alpha_{c}$ depends on $n_{b}^{\mathrm{c}}$ with the relation

$$
\alpha_{c}\left(n_{b}^{\mathrm{c}}\right)= \begin{cases}-\frac{1.334}{x}+1 & n_{b}^{\mathrm{c}}>1 \\ \frac{1}{3} & n_{b}^{\mathrm{c}}=1\end{cases}
$$

obtained from a parameter fit of the optimization results. For comparison, the curves for $\alpha_{c}$ and $\alpha_{d}$ as introduced in eq. (4) and (6), respectively, are given. It is obvious that the optimizer yields significantly 
different results. Furthermore, it is interesting to note that the optimum of $\alpha_{c}$ and $\alpha_{d}$ is 1 for both factors for large $n_{b}^{\mathrm{c}}$ as one would expect for a strict surface coupling - which is indeed the limit case for $n_{b}^{\mathrm{c}} \rightarrow \infty$. In contrast, the choice of $\alpha_{d}=\frac{1}{2}$ according to eq. (6) does not cover this expectation.

Usually, the motivation for such multiscale approaches is to reduce computational by introducing a coarse resolution while maintaining the fine-scale accuracy where it is needed. Therefore, we strive to maximize the difference between the resolutions, resulting in weighting factors of $\alpha_{d}=1$ and $\alpha_{c} \rightarrow 1$ for the coupling scheme presented here.

So far, we have only considered $k^{\mathrm{nn}} / k^{\mathrm{sn}}=1$, which is why we are now evaluating the method's performance for other stiffness ratios. To this end, we examine the same system as above $\left(n_{A}^{\mathrm{d}}=10, n_{P}^{\mathrm{c}}=\right.$ $\left.1, n_{\mathrm{FE}}^{\mathrm{c}}=4\right)$ with $n_{b}^{\mathrm{c}}=10$, and vary only the ratio of next-neighbor to second-to-next-neighbor interactions $k^{\mathrm{nn}} / k^{\mathrm{sn}}$. It is evident from Fig. 5 , that our coupling method is only working well for $k^{\mathrm{nn}} / k^{\mathrm{sn}} \geq 2$, as the error increases significantly for smaller $k^{\mathrm{nn}} / k^{\mathrm{sn}}$.
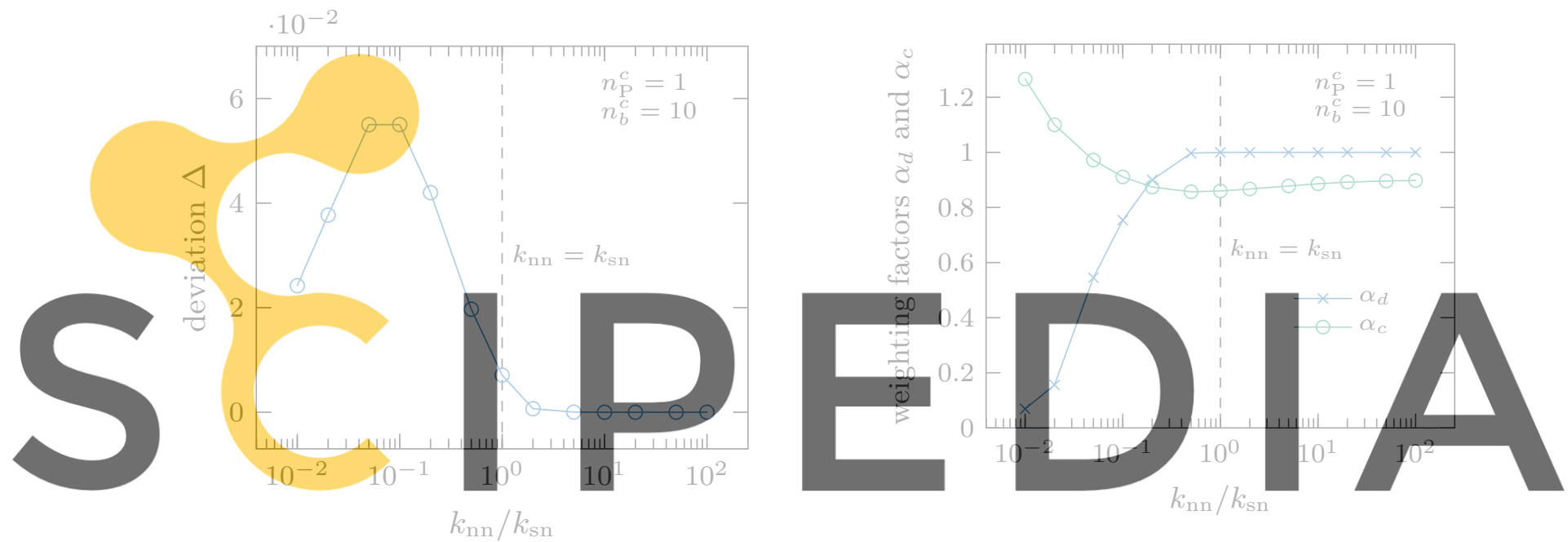

Register for free at https//www.scipedia.com to downjoads:theuversionthe withould the iwatermark

Figure 5: Influence of the ratio of local to nonlocal interactions $k^{\mathrm{nn}} / k^{\mathrm{sn}}$ on the deviation $\Delta$ for $n_{P}^{\mathrm{c}}=1$ and $n_{b}^{\mathrm{c}}=10$.

cal interactions $k^{\mathrm{nn}} / k^{\mathrm{sn}}$ on the optimized weighting factors $\alpha_{d}$ and $\alpha_{c}$ for $n_{P}^{c}=1$ and $n_{b}^{c}=10$.

The associated weighting factors illustrate this in Fig. 6 . At $k^{\mathrm{nn}} / k^{\mathrm{sn}} \leq 0.5, \alpha_{d}$ and $\alpha_{c}$ deviate significantly from the previously observed behaviors: $\alpha_{d}$ seems to converge towards 0 , while $\alpha_{c}$ increases and reaches values above 1 for very small $k^{\mathrm{nn}} / k^{\mathrm{sn}}$. If $k^{\mathrm{sn}}$ is significantly larger than $k^{\mathrm{nn}}$, i.e., the material behavior is dominated by non-local interactions, the optimizers fail to identify a set of weighting factors to account for that. This is plausible since continuum mechanics is a local method, which we expect to struggle for such material. However, this is not a major problem in the context of polymers since their covalent bonds are usually prevailing their long range interactions, e.g., van der Waal's forces.

\section{CONCLUSION}

In this contribution, we discuss a method for bridging the scale between continuum and particle-based descriptions separated by an interface instead of a handshake region as used in other approaches. We 
introduce padding atoms, representing the continuum, to provide the missing interaction partners for the particles adjacent to the interface. By performing a simple 1D study we demonstrate how the coupling parameters can be optimized and find that this concept performs best for small coupling regions, large differences in the scales, and materials with dominant next-neighbor interactions. Consequently, the method presented here seems suitable for the multiscale modeling of amorphous polymers.

In the future, we aim to expand this approach to 3D and evaluate whether our findings, especially for the optimal weighting factors, still hold.
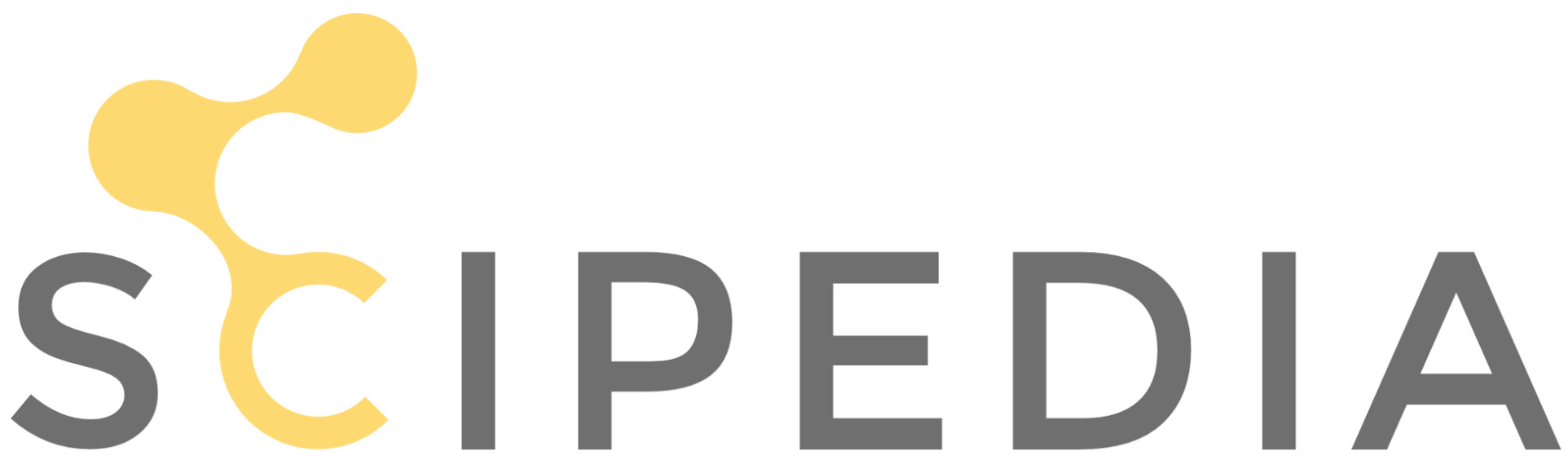

Register for free at https//www.scipedia.com to download the version without the watermark 


\section{REFERENCES}

[1] Zhao, W.; Ries, M.; Steinmann, P. \& Pfaller, S.; A viscoelastic constitutive model for polymers based on molecular dynamics simulation under finite uniaxial deformation, submitted.

[2] Ries, M.; Possart, G.; Steinmann, P. \& Pfaller, S.; Extensive CMD Simulations of Atactic PS Providing Pseudo Experimental Data to Calibrate Nonlinear Inelastic Continuum Mechanical Constitutive Laws, Polymers, Multidisciplinary Digital Publishing Institute, 2019 , 11 , 1824.

[3] Ries, M.; Steinmann, P. \& Pfaller, S.; Characterization of Polystyrene under Shear Deformation Using Molecular Dynamics, Developments and Novel Approaches in Nonlinear Solid Body Mechanics, Springer, 2020 , 219-229.

[4] Tadmor, E. B.; Ortiz, M. \& Phillips, R.; Quasicontinuum analysis of defects in solids, Philosophical Magazine A, 1996, 73 , 1529-1563.

[5] Tadmor, E. B.; Phillips, R. \& Ortiz, M.; Mixed Atomistic and Continuum Models of Deformation in Solids, Langmuir, 1996, 12, 4529-4534.

[6] Ben Dhia, H. \& Rateau, G.; The Arlequin method as a flexible engineering design tool, International Journal for Numerical Methods in Engineering, Wiley Blackwell (John Wiley \& Sons), 2005 , 62 , $1442-1462$.

[7] Ben Dhia, H.; Elkhodja, N. \& Roux, F. X.; Multimodeling of multi-alterated structures in the Arlequin framework. Solution with a Domain-Decomposition solver, European Journal of Compu-

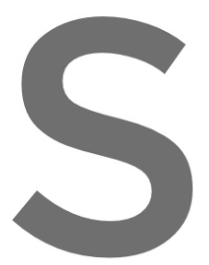
tational Mechanic

[8] Belytschko, T. \& national Journal for

[9] Guidault, P.-A. \& B composition method using Lagrange multiplias

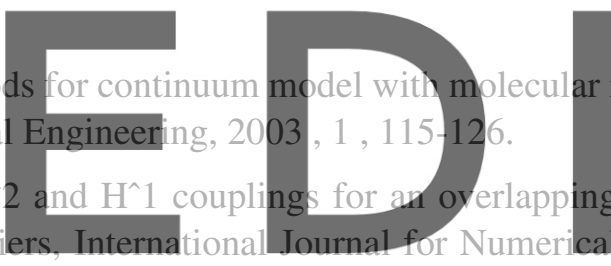
model, Inter domain deEngineering, Wiley Blackwell (John Wiley \& Sons), 2007, 70 , 322-350.

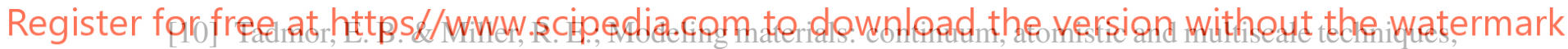
Cambridge University Press, 2011.

[11] Pfaller, S.; Multiscale Simulation of Polymers, Friedrich-Alexander-Universität ErlangenNürnberg, Friedrich-Alexander-Universität Erlangen-Nürnberg, 2015.

[12] Rahimi, M.; Karimi-Varzaneh, H. A.; Böhm, M. C.; Müller-Plathe, F.; Pfaller, S.; Possart, G. \& Steinmann, P.; Nonperiodic stochastic boundary conditions for molecular dynamics simulations of materials embedded into a continuum mechanics domain, Journal of Chemical Physics, 2011, 134 , 154108 .

[13] Pfaller, S.; Possart, G.; Steinmann, P.; Rahimi, M.; Müller-Plathe, F. \& Böhm, M. C.; A comparison of staggered solution schemes for coupled particle-continuum systems modeled with the Arlequin method, Computational Mechanics, Springer-Verlag, 2012 , 49 , 565-579.

[14] Pfaller, S.; Rahimi, M.; Possart, G.; Steinmann, P.; Müller-Plathe, F. \& Böhm, M.; An Arlequinbased method to couple molecular dynamics and finite element simulations of amorphous polymers and nanocomposites, Computer Methods in Applied Mechanics and Engineering, 2013 , 260 , 109- 
129.

[15] Pfaller, S.; Kergaßner, A. \& Steinmann, P.; Optimisation of the Capriccio Method to Couple Particle- and Continuum-Based Simulations of Polymers, Multiscale Science and Engineering, $2019,1,318-333$.

[16] Zhao, W.; Steinmann, P.; \& Pfaller, S.; A particle-continuum coupling method for multiscale simulations of viscoelastic-viscoplastic amorphous glassy polymers, submitted.

[17] Pfaller, S.; Possart, G.; Steinmann, P.; Rahimi, M.; Müller-Plathe, F. \& Böhm, M.; Investigation of interphase Effects in Silica-Polystyrene Nanocomposites Based on a Hybrid Molecular-DynamicsFinite-Element Simulation Framework, Physical Review E, 2016 , 93 , 052505.

[18] Ries, M.; Possart, G.; Steinmann, P.; \& Pfaller, S.; A coupled MD-FE methodology to characterize mechanical interphases in polymeric nanocomposites, in preparation.

[19] Lagarias, J. C., Reeds, J. A., Wright, M. H., \& Wright, P. E.; Convergence properties of the NelderMead simplex method in low dimensions SIAM Journal on optimization, 1998, 9.1, 112-147.

[20] Coleman, T. F., \& Li, Y.; An interior trust region approach for nonlinear minimization subject to bounds SIAM Journal on optimization, 1996 , 6(2), 418-445.
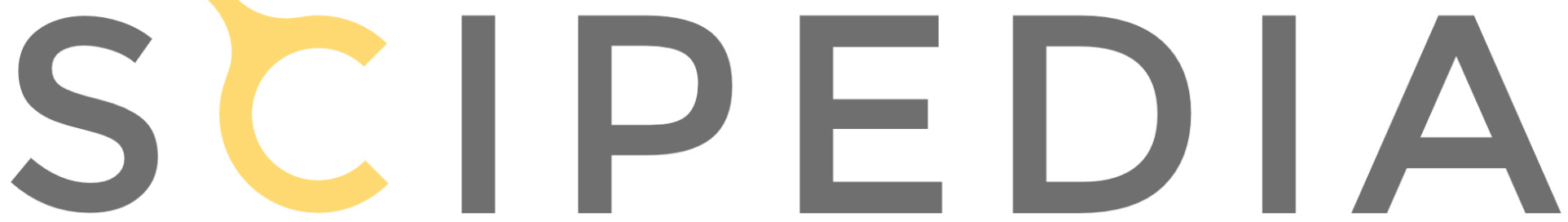

Register for free at https//www.scipedia.com to download the version without the watermark 